\title{
In-situ Observation of Catalytic Reaction in Gas Atmosphere Using an Aberration Corrected STEM
}

\author{
Manabu Shirai ${ }^{1}$, Akinari Hanawa ${ }^{1}$, Hideki Kikuchi ${ }^{1}$, Hiromi Inada ${ }^{2}$ and Hiroaki Matsumoto ${ }^{3}$
}

1. Science System Design Division, Hitachi High-Technologies Corporation, Hitachinaka, Ibaraki, Japan

2. Science Systems Sales \& Marketing Division, Hitachi High-Technologies, Nishishinbashi, Tokyo, Japan

${ }^{3 .}$ Electron Microscope \& AFM Systems Sales Department, China Business Operations, Hitachi HighTechnologies Beijing Branch, Chao Yang District, Beijing, China

Understanding the evolution of functional materials is highly desired for development and improvement of nanomaterials. In-situ TEM observation is powerful technique for studying the behavior of the materials under various condition such as heating, cooling, and various gas atmosphere in nano meter scale. The authors have developed in situ observation technique based on a cold FE TEM with capabilities of simultaneous SEM and STEM images and applied it to study the degrading mechanism of electrocatalysis under air at $200{ }^{\circ} \mathrm{C}$ [1][2]. Advancing miniaturization of research target makes imaging with sub angstrom resolution essential. Recent development of the aberration corrector enable us to observe samples at atomic resolution. Furthermore, micro electro mechanical systems (MEMS) chipbased specimen holders were developed. These holders provide highly stable observation even at high temperature. In this study, in-situ observation of catalysis under gas atmosphere using an aberration corrected STEM with a MEMS heating holder is reported.

In-situ observation was performed with an HF5000 $200 \mathrm{kV}$ TEM/STEM (Hitachi High-Technologies Co.) equipped with a probe corrector. Figure 1 shows the schematic diagram of differential pumping system of the HF5000 for environmental (S)TEM imaging. It is not a customized model but based on a standard one. An orifice is added between a gun valve and a specimen chamber to strengthen differential pumping system. The gas is introduced into the specimen chamber via a gas injection nozzle installed near the specimen chamber. Maximum pressure at sample is about $10 \mathrm{~Pa}$. Figure 2 shows annular darkfield (ADF) STEM images of $\mathrm{Si}(110)$ after $\mathrm{H}_{2}$ and $\mathrm{O}_{2}$ gas injection up to $2 \mathrm{~Pa}$ at room temperature. Silicon dumbbells were observed as clearly separated bright dots in the STEM images, demonstrating atomic-resolution performance without notable scattering effect by gas molecules. A MEMS-based holder (Blaze, Hitachi High-Technologies Canada, Inc.) was employed for in-situ heating observation. A $30 \mathrm{~nm}$ thick SiN support film for the sample area provides electron transparency and high stability.

Reduction reaction experiment of $\mathrm{NiAu}$ particles were tried with this system. Figure 3 shows ADFSTEM and SEM images of the NiAu particles before and after $\mathrm{CO}_{2}$ and $\mathrm{H}_{2}$ mixed gas injection at various temperature. In the initial state, the particles are in the "dumbbell" structure which a Ni particle binds to an Au particle. Contrast of the particles in the ADF-STEM become uniform under mixed gas atmosphere at $600{ }^{\circ} \mathrm{C}$, suggesting the Au particles gradually merged into the Ni sphere and formed $\mathrm{NiAu}$ alloy. Edge contrast of some particles in ADF-STEM was enhanced after cooling down to $300{ }^{\circ} \mathrm{C}$. This result suggests that the alloy particles were changed to core-shell structure which the Ni core are covered with $\mathrm{Au}$ shell. In the presentation, analytical details of this phenomena using high resolution imaging, EDX and EELS analyses will be introduced. 


\section{References:}

[1] H. Matsumoto et al., Microscopy and Analysis 27(7) (2013) 11.

[2] H. Matsumoto et al., Microsc. Microanal. 27 (2013) 11.

[3] The authors acknowledge to Prof. S. Miao of Dalian Institute of Chemical Physics Chinese Academy of Sciences for providing the catalyst samples and giving his fruitful comments.

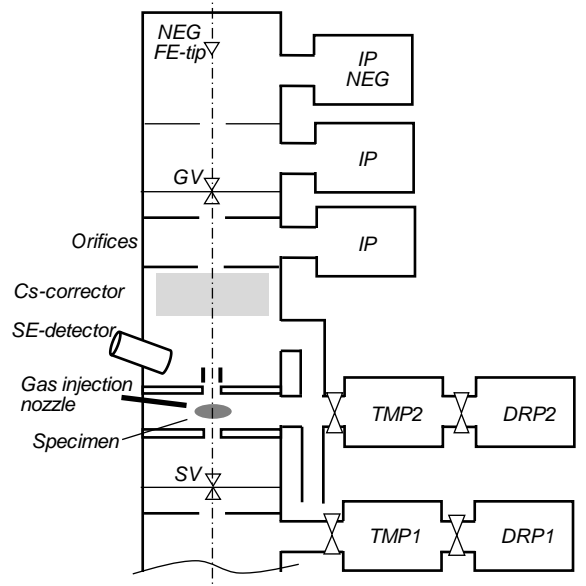

Figure 1. Schematic diagram of differential pumping system modified for environmental (S)TEM imaging.
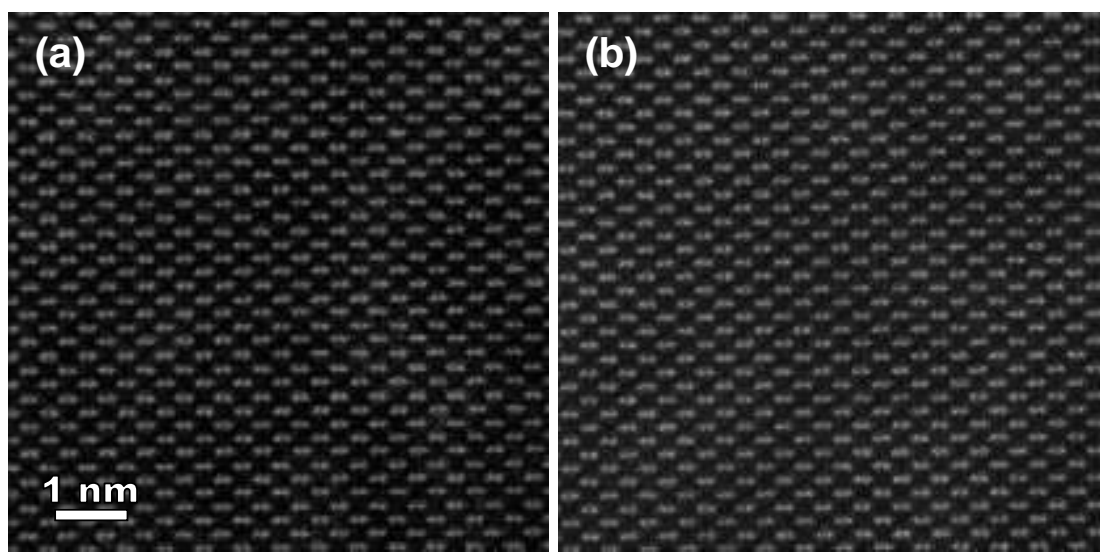

Figure 2. ADF-STEM images of $\mathrm{Si}(110)$ after $\mathrm{H}_{2}$ and $\mathrm{O}_{2}$ gas injection up to $2 \mathrm{~Pa}$. (a) $\mathrm{H}_{2}$ (b) $\mathrm{O}_{2}$ (a) R.T.

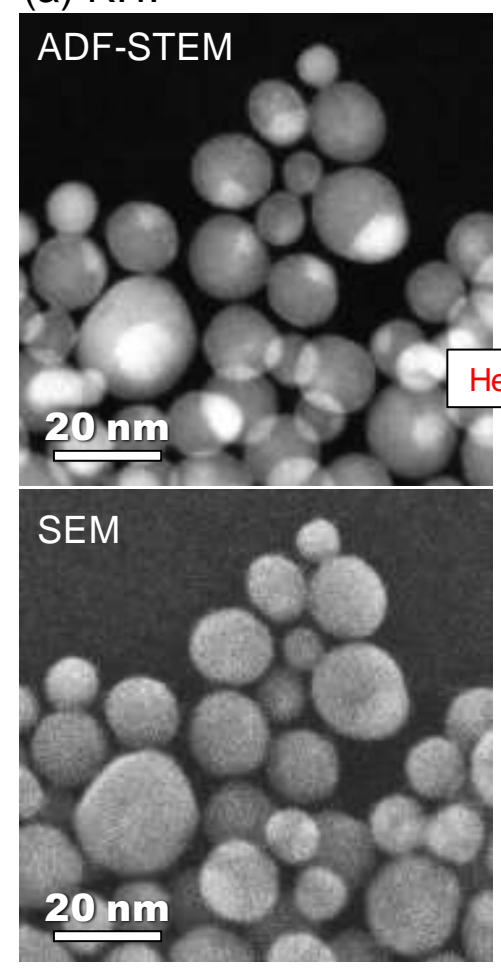

(b) $600^{\circ} \mathrm{C}, \mathrm{CO}_{2}+\mathrm{H}_{2} 2 \mathrm{~Pa}$

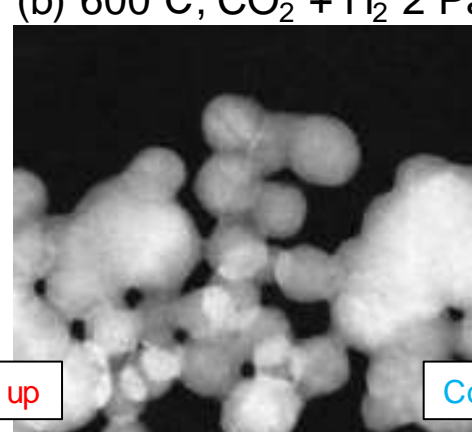

Cooling down
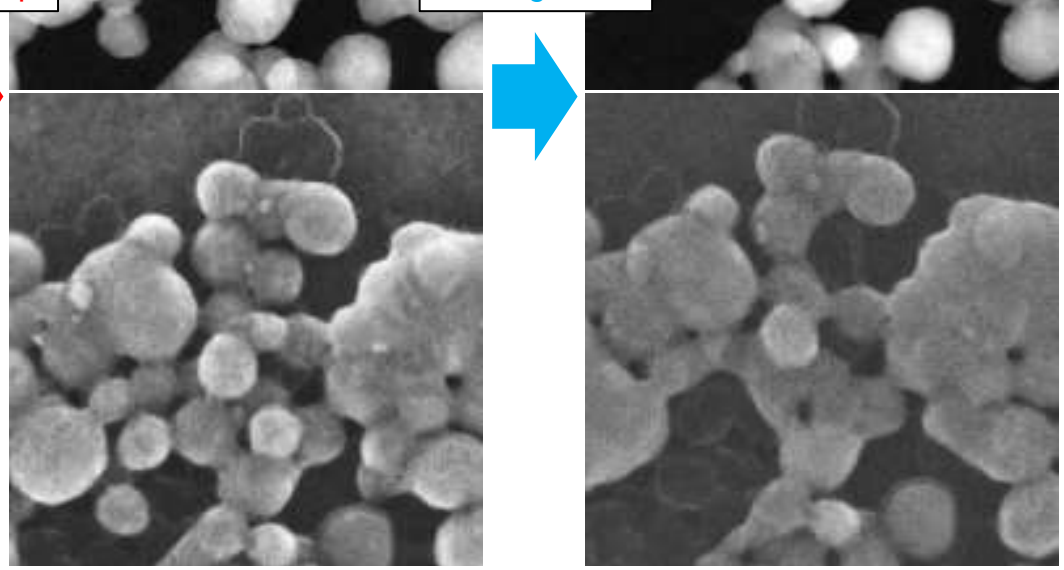

Figure 3. ADF-STEM and SEM images of NiAu particles during reduction reaction.

(a) under vacuum at R.T. (b) under $\mathrm{CO}_{2}$ and $\mathrm{H}_{2}$ at $600^{\circ} \mathrm{C}$ (c) under $\mathrm{CO}_{2}$ and $\mathrm{H}_{2}$ at $300^{\circ} \mathrm{C}$ 